\title{
Optimizing hematopoietic recovery following bone marrow transplantation
}

Commentary

See related article, pages $1579-1585$.

\author{
Ronald Paquette ${ }^{1,2}$ and Kenneth Dorshkind ${ }^{2,3}$ \\ ${ }^{1}$ Division of Hematology/Oncology, Department of Medicine, \\ ${ }^{2}$ The Hematopoietic Malignancies and Bone Marrow Transplantation Program, Jonsson Comprehensive Cancer Center, and \\ ${ }^{3}$ Department of Pathology and Laboratory Medicine, University of California Los Angeles School of Medicine, Los Angeles, California, USA \\ Address correspondence to: Kenneth Dorshkind, Department of Pathology and Laboratory Medicine 173216, \\ UCLA School of Medicine, 10833 Le Conte Avenue, Los Angeles, California 90095-1732, USA. \\ Phone: (310) 206-9535; Fax: (310) 206-9391; E-mail: kdorshki@mednet.ucla.edu.
}

J. Clin. Invest. 109:1527-1528 (2002). doi:10.1172/JCI200215916.

Blood cell production is a dynamic process in which immature precursors progress through a series of developmental events that culminate in the production of mature cells of the erythroid, myeloid, and lymphoid lineages. The characteristics of cells at particular stages of development and the intrinsic and extrinsic signals that regulate their growth and differentiation are becoming increasingly well defined. As a result, it is now possible to orient them in the hierarchical model shown in Figure 1 (1).

At the head of this schema is the pluripotent hematopoietic stem cell (PHSC), which can self-renew and generate differentiated progeny (2). In both humans and mice, PHSCs lack cell surface determinants expressed by committed myeloid and lymphoid lineage cells. Further resolution of this lineage-negative population based on the expression of Thy- 1 and Sca- 1 in the mouse (3) and CD34 in humans (4) has allowed PHSCs to be isolated from the bone marrow. This achievement, made possible by advances in $\mathrm{mAb}$ production and flow cytometry, is remarkable because PHSCs account for only $0.05-0.1 \%$ of total bone marrow nucleated cells. PHSC progeny can also be purified from murine bone marrow based on their phenotypic characteristics. These include the common lymphoid progenitor (CLP), from which $B$, $\mathrm{T}$, and natural killer cells descend, and the common myeloid progenitor (CMP), from which all myeloid and erythroid cells are derived $(5,6)$.

That the populations designated as PHSCs, CMPs, and CLPs have the developmental potential ascribed to them was determined prospectively: Cells expressing particular phenotypes were isolated, and their ability to generate various hematopoietic lineages was determined in vitro and in vivo. These types of reconstitution studies revealed that only PHSCs can mediate stable, long-term hematopoietic repopulation of recipients; more differentiated progenitors provide transient reconstitution. The ability to isolate and transplant PHSCs has a number of clinical advantages. For example, one complication following allogeneic bone marrow transplantation is T cell-mediated graft-versushost disease. Transplantation of PHSC-enriched, T cell-depleted donor

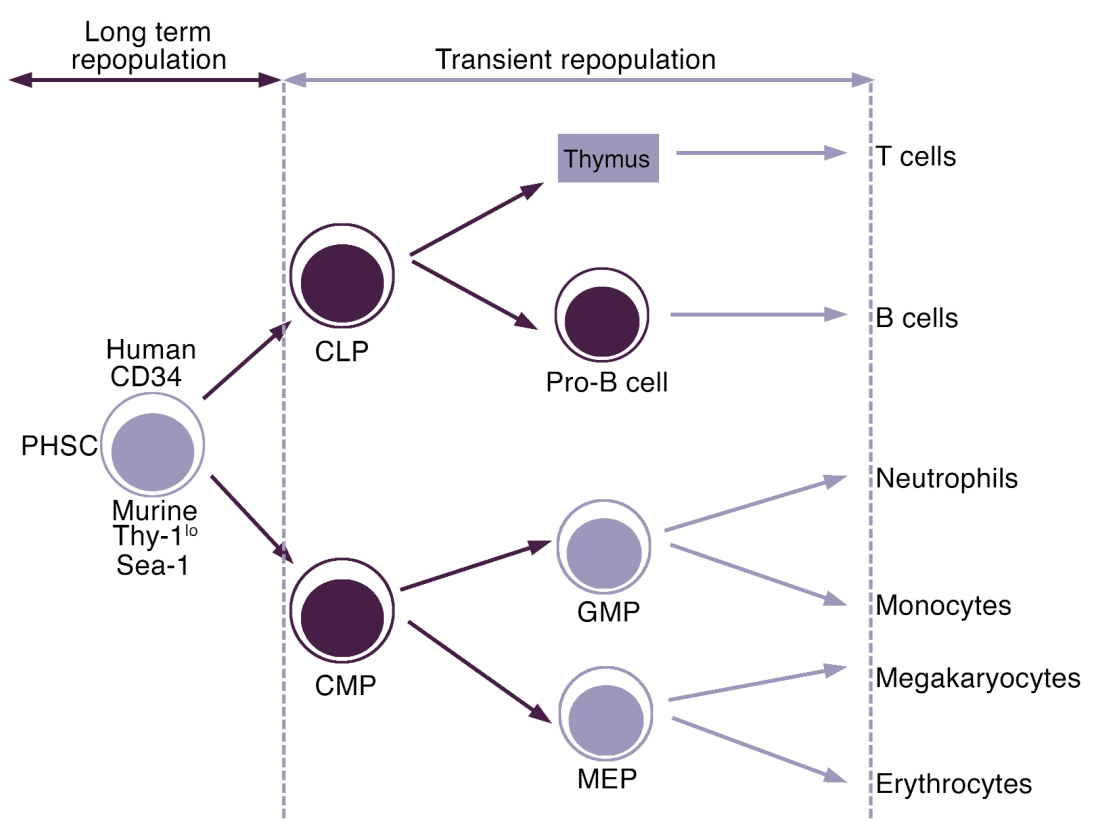

Figure 1

The hematopoietic hierarchy. The figure depicts selected cell surface determinants used to isolate PHSCs. Common myeloid progenitors (CMPs) and common lymphoid progenitors (CLPs) can also be defined in the mouse. The figure shows that only PHSCs provide long-term, stable repopulation of recipients, while other cells mediate different degrees of transient reconstitution. The radioprotective megakaryocyte/erythroid progenitor in the mouse is indicated. 
hematologists circumvent this problem by supplementing PHSCs with a small amount of unfractionated bone marrow, which contains progenitors that can rapidly generate myeloid and erythroid progeny. The transient reconstitution mediated by the more differentiated cells allows the mice to survive the interval between the conditioning regimen and the stable reconstitution of blood cells by transplanted or endogenous PHSCs.

The precise identity of the radioprotective bone marrow cells has been a mystery. Lymphocytes were not likely to be required, because mice and humans can live, albeit in a restricted manner, without them. More likely candidates for the radioprotective population include the progenitors of granulocytes and monocyte/macrophages, which provide the first line of defense in many infections, or of other crucial cell types, such as erythrocytes or platelets.

The work by $\mathrm{Na}$ Nakorn et al. in this issue of the JCI (7) now helps to resolve the remaining uncertainties about the identity of this key population. Using highly sophisticated cell purification protocols, these investigators isolated CMPs, megakaryocyte/erythrocyte-restricted progenitors (MEPs), and granulocyte/monocyte-restricted progenitors (GMPs) and transplanted them into lethally irradiated recipients. Transplanted CMPs gave rise to granulocytes, monocytes, megakaryocytes, and erythroid cells and allowed survival of the recipients. However, when MEPs and GMPs were compared, only the former conferred radioprotection, suggesting that thrombopoiesis and erythropoiesis were more critical than myelopoiesis in rescuing the mice from the lethal effects of radiation.

These data suggest that administration of selected hematopoietic cells, such as MEPs, to patients who have received pretransplant conditioning chemo- and/or radiotherapy might be of value. In fact, such approaches are being actively pursued. Several groups recently reported that administration of ex vivo expanded cells, generated by culturing hematopoietic progenitors in a limited number of hematopoietic growth factors for 9-10 days, along with unmanipulated peripheral blood progenitor cells reduced posttransplantation neutropenia, thrombocytopenia, and anemia (8-11). The ex vivo expanded populations were heterogeneous, and the cells that contributed to the accelerated hematologic recovery were not identified. These clinical observations, combined with the data reported by $\mathrm{Na}$ Nakorn et al. (7), suggest that selected hematopoietic progenitor cell populations will be useful therapeutically in patients who have received myeloablative treatments. Further refinements of this technique will require a still more precise characterization of the progenitor cell population(s) that reconstitutes critical hematopoietic cells following transplantation.

1. Dexter, T.M. 1990. Introduction to the haemopoietic system. Cancer Surv. 9:1-5.

2. Phillips, R.L., et al. 2000. The genetic program of hematopoietic stem cells. Science. 288:1635-1640.

3. Spangrude, G.J., Heimfeld, S., and Weissman, I.L. 1988. Purification and characterization of mouse hematopoietic stem cells. Science. 241:58-62.

4. Kim, H., Whartenby, K.A., Georgantas, R.W., III, Wingard, J., and Civin, C.I. 2002. Human CD34(+) hematopoietic stem/progenitor cells express high levels of FLIP and are resistant to Fas-mediated apoptosis. Stem Cells. 20:174-182.

5. Kondo, M., Weissman, I., and Akashi, K. 1997. Identification of clonogenic common lymphoid progenitors in mouse bone marrow. Cell. 91:661-672

6. Akashi, K., Taver, D., Miyamoto, T., and Weissman, I. 2000. A clonogenic common myeloid progenitor that gives rise to all myeloid lineages. Nature. 404:193-197.

7. Na Nakorn, T., Traver, D., Weissman, I.L., and Akashi, K. 2002. Myeloerythroid-restricted progenitors are sufficient to confer radioprotection and provide the majority of day $8 \mathrm{CFU}-\mathrm{S}$. J. Clin. Invest. 109:1579-1585. doi:10.1172/JCI200215272.

8. Reiffers, J., et al. 1999. Abrogation of post-myeloablative chemotherapy neutropenia by ex-vivo expanded autologous CD34-positive cells. Lancet. 354:1092-1093.

9. Paquette, R.L., et al. 2000. Ex vivo expanded un selected peripheral blood progenitor cells reduce posttransplantation neutropenia, thrombocytopenia and anemia in patients with breast can cer. Blood. 96:2385-2390.

10. McNiece, I., et al. 2000. Ex vivo expanded periph eral blood progenitor cells provide rapid neutrophil recovery after high-dose chemotherapy in patients with breast cancer. Blood. 96:3001-3007.

11. Paquette, R.L., et al. 2002. Culture conditions affect the ability of ex vivo expanded peripheral blood progenitor cells to accelerate hematopoietic recovery. Exp. Hematol. 30:374-380. 